\section{Dor lombar em usuários de um plano de saúde: prevalência e fatores associados}

\author{
Lower back pain in health insurance policyholders: \\ prevalence and associated factors
}

\author{
1 Programa de Pós-gradu- \\ ação em Saúde Coletiva \\ Universidade do Vale do Rio \\ dos Sinos, \\ São Leopoldo, Brasil. \\ 2 Faculdade de Medicina \\ Universidade Federal de \\ Pelotas, Pelotas, Brasil. \\ Correspondência \\ J. S. Dias-da-Costa \\ Departamento de Medicina \\ Social, Faculdade de \\ Medicina, Universidade \\ Federal de Pelotas. \\ Av. Duque de Caxias 250, \\ Pelotas, RS \\ 96030 002, Brasil. \\ jcosta@epidemio-ufpel.org.br
}

\begin{abstract}
A cross-sectional study was conducted with individuals of both sexes (20-59 years of age), all covered by the COOPERSINOS Health Care Plan of Vale do Rio dos Sinos University. The study aimed to determine the prevalence of lower back pain and associated factors in the previous three months. A self-applied questionnaire included socioeconomic, demographic, occupational, and lifestyle questions. Prevalence of lower back pain among the 775 individuals was $52.8 \%$ in the previous year (95\%CI: 49.3-56.3) and 46\% in the previous three months (95\%CI: 42.5-49.5). Poisson regression for lower back pain in the previous 90 days showed an association with: (1) age 40-49 years; (2) self-reported strenuous or difficult work; and (3) smoking. Despite the high prevalence of lower back pain and difficulty in performing work activities, the pain was not disabling. Although 29.9\% of workers reported some difficulty in performing their daily or work-related activities due to lower back pain, absenteeism was quite low.
\end{abstract}

Low Back Pain; Prepaid Health Plans; Prevalence

\author{
Mauro Gomes Matos 1 \\ Élida Azevedo Hennington 1 \\ Ana Lúcia Hoefel 1 \\ Juvenal Soares Dias-da-Costa 1,2
}

\section{Introdução}

A dor lombar é uma das mais comuns afecções músculo-esqueléticas e sua importância pode ser constatada pelas medidas de incidência e prevalência na população geral de adultos e em comunidades de trabalhadores 1,2,3. Deve-se também observar sua participação nas causas de absenteísmo ao trabalho, de incapacidade temporária ou permanente e mesmo de invalidez; bem como o seu custo econômico sobre os sistemas de seguridade social 4 .

Segundo Deyo 5, a dor na coluna lombar atinge níveis epidêmicos na população em geral e Andersson 6 relata que esta é uma afecção muito comum na população, atingindo prevalência de $70 \%$ em países industrializados. A dor lombar é uma importante causa de incapacidade, ocorrendo em prevalências elevadas em todas as culturas, influenciando a qualidade de vida das pessoas 7. Estudos têm demonstrado que $60 \%$ a $80 \%$ da população adulta têm ou tiveram um episódio de dor incapacitante na coluna vertebral, principalmente na região lombar 8,9,10, ou manifestou dor lombar em algum momento de suas vidas independente da ocupação 11,12 . Cerca de $80 \%$ da população já tiveram algum episódio, cuja intensidade pode variar de um leve desconforto até dores incapacitantes e de longa duração 13. Papageorgiou et al. 14 realizaram estudo transversal populacional sobre dor lombar no mês numa população 
de adultos do Reino Unido, encontrando prevalência de $39 \%$.

Alguns autores reconhecem a etiologia da dor lombar como multifatorial, incluindo fatores sócio-econômicos e demográficos, estilo de vida urbano sedentário, obesidade, fumo, posturas viciosas durante o trabalho, aumento da sobrevida média da população e outros 15,16. Inúmeros estudos epidemiológicos buscam a relação de dor lombar com exigências físicas do trabalho e fatores ergonômicos como o levantamento de cargas, flexões e torções do tronco, vibrações e esforços repetitivos 17,18,19. O presente artigo objetivou estudar a freqüência e fatores associados de dor lombar nos últimos três meses entre trabalhadores associados na Cooperativa do Vale do Rio dos Sinos (COOPERSINOS), cooperativa formada por funcionários da Universidade do Vale do Rio dos Sinos (UNISINOS).

\section{Métodos}

Foi realizado um estudo transversal entre outubro de 2005 e maio de 2006, que através de censo investigou diversas características dos titulares do plano de saúde da COOPERSINOS.

A população do presente estudo foi constituída de adultos (20 a 59 anos) titulares do plano de saúde, que em setembro de 2005 foi estimada em 1.108 sujeitos. Deste grupo, 226 adultos titulares do plano foram excluídos (oito por licença maternidade e/ou licença para estudos, 61 por demissões e 157 usuários titulares classificados como externos, ou seja, que não trabalhavam mais na universidade, mas continuavam vinculados ao plano de saúde ou realizavam as suas atividades de trabalho externamente ao campus). Ao final da coleta de dados, houve 107 (12,1\%) perdas e recusas. Contabilizaram-se 27 recusas na participação e 80 perdas pela não localização dos usuários. Assim sendo, este estudo contou com $88 \%$ de taxa de participação, ou seja, 775 usuários adultos titulares do plano de saúde do total de 882 usuários elegíveis.

Antes do início da coleta de dados os usuários do plano de saúde selecionados foram informados através de correspondência sobre a pesquisa e a importância da participação de todos, além da garantia de sigilo da identidade dos informantes. A maior parte das entrevistas era marcada por telefone. Para se reduzir o número de perdas e recusas foi enviada uma segunda carta para sensibilizar aqueles que não estavam sendo encontrados.

As informações foram coletadas por meio de dois questionários auto-aplicados e pré-codificados (questionário individual de saúde e sócio-econômico da família) preenchidos pelos próprios indivíduos, com ajuda, se necessário, de bolsistas previamente treinados. A codificação foi realizada pelos bolsistas logo após o preenchimento dos questionários e revisados pelos pesquisadores.

A presença de dor lombar foi estabelecida através da identificação da região lombar na coluna vertebral (por meio de uma figura de pessoa em posição ereta e dorsal com as regiões: lombar, torácica e cervical destacada) como um local de dor ou desconforto nos últimos três meses, adaptado de Kourinka et al. 20. Esse instrumento tem sido utilizado internacionalmente, tendo sido desenvolvido para padronizar pesquisas sobre investigações músculo-esqueléticas e já foi validado na cultura brasileira 21 .

Foram exploradas as associações entre dor lombar nos últimos três meses com variáveis demográficas, sócio-econômicas, ocupacionais e hábitos de vida. As variáveis demográficas incluídas na análise foram: idade, cor da pele declarada pelos respondentes e se vivia com companheiro. Foram consideradas como variáveis sócio-econômicas: classificação econômica, escolaridade e renda familiar per capita. As categorias de classes econômicas foram definidas com base na tabela de classificação econômica da Associação Brasileira de Empresas de Pesquisas (ABEP; http:// www.anep.org.br/codigosguias/ABEP_CCEB. pdf, acessado em 10/Mai/2006), estabelecida no Critério de Classificação Econômica Brasil, a partir da posse de bens materiais, da escolaridade do chefe da família e da presença de empregados domésticos nas residências.

Investigaram-se algumas variáveis relacionadas ao trabalho, tais como carga diária no trabalho, trabalho classificado como penoso, esforço no trabalho e posição adotada no trabalho. A carga diária despendida no trabalho correspondeu ao número de horas trabalhadas, o trabalho foi classificado como sendo ou não diário e o esforço realizado como muito leve/leve ou pesado/muito pesado. Em relação à categoria no trabalho, foram classificados dois grupos de trabalhadores: (i) professores, pesquisadores e profissionais liberais, e (ii) técnico-administrativos. Investigou-se a posição adotada durante a maior parte do tempo no seu trabalho (em pé parado, caminhando e sentado) e para cada questão ofereceram-se as opções nunca, raramente, freqüentemente ou sempre 18.

Entre os hábitos de vida foram analisadas as seguintes variáveis: tabagismo, estado nutricional e atividade física. Verificou-se o estado nutricional através do cálculo do índice de massa corporal (peso/altura 2 ; IMC $=\mathrm{kg} / \mathrm{m}^{2}$ ), sendo consideradas com sobrepeso os respondentes 
com IMC entre $25,0 \mathrm{~kg} / \mathrm{m}^{2}$ e $29,9 \mathrm{~kg} / \mathrm{m}^{2}$ e obesos com $30,0 \mathrm{~kg} / \mathrm{m}^{2}$ ou mais 22 . Foi perguntado se os indivíduos praticavam atividade física no lazer e com que freqüência (duração e número de vezes por semana), sendo considerados ativos os participantes que realizavam atividade física por pelo menos três vezes na semana e com duração mínima de trinta minutos por sessão 23.

A entrada dos dados foi realizada através do programa Epi Info (Centers for Disease Control and Prevention, Atlanta, Estados Unidos), em dupla entrada e posterior comparação, para se eliminar erros de digitação. A análise bruta dos dados foi realizada através do programa SPSS, versão 11.0 (SPSS Inc., Chicago, Estados Unidos). A análise procurou verificar a associação das variáveis demográficas, sócio-econômicas, ocupacionais e hábitos de vida com dor lombar nos últimos três meses 24 . A análise ajustada para controle dos fatores de confusão foi realizada no programa Stata (Stata Corp., College Station, Estados Unidos) e seguiu modelo hierarquizado 25 com dois níveis de variáveis. Esse modelo pressupõe uma ordem hierarquizada das variáveis determinando o desfecho. Ingressaram na análise todas as variáveis que, na análise bivariada, apresentaram valor de p de até 0,2. Assim, no primeiro nível do modelo ingressaram as variáveis demográficas e sócio-econômicas ajustadas entre si e determinantes do nível imediatamente inferior constituído pelos hábitos de vida e pelas variáveis relacionadas ao trabalho. Todas determinando dor lombar nos últimos três meses. A análise multivariável foi realizada através de regressão de Poisson e os resultados expressos como razões de prevalência 26 .

O projeto de pesquisa foi aprovado pelo Comitê de Ética em Pesquisa da UNISINOS e todos os participantes assinaram Termo de Consentimento Livre e Esclarecido.

\section{Resultados}

Entre as 775 pessoas entrevistadas, 355 (46\%; IC95\%: 42,5-49,5) referiram dor lombar nos últimos três meses. A prevalência de dor referida pela população na coluna vertebral no último ano foi de 71,5\% (IC95\%: 68,3-74,7), sendo que a região mais acometida foi a coluna lombar, representando uma prevalência de 52,8\% ano (IC95\%: 49,3-56,3). Constatou-se que a freqüência de dor lombar crônica (dor por um período igual ou superior a seis semanas) foi de 3,8\% (IC95\%: $2,4-5,1)$, a dor lombar que motivou procura de consulta médica foi de 6,9\% (IC95\%: 5,1-8,7) e a dor com ausência ao trabalho foi de $0,4 \%$ (IC95\%: 0,2-0,5). A prevalência da combinação de dor lombar nos últimos três meses e/ou dor crônica, e/ou com consulta médica, e/ou com ausência ao trabalho foi de 9,5\% (IC95\%: 7,4-11,6).

Dentre os indivíduos que tiveram dor lombar nos últimos três meses, verificou-se que 29,9\% relataram ter tido dificuldade para realizar as suas atividades de vida diária ou no trabalho por causa de dor lombar nos últimos três meses. Deve-se destacar que, $1,7 \%$ das pessoas investigadas foram hospitalizadas e $0,5 \%$ tiveram que mudar de trabalho devido à dor lombar.

Entre os 775 indivíduos entrevistados observou-se que o maior percentual de adultos era do sexo feminino $(54,2 \%)$ e pertencia à faixa etária entre 30 e 49 anos $(64,4 \%)$ (Tabela 1). A maioria era de cor branca $(94,9 \%)$ e vivia com companheiro (59,5\%). Mais da metade encontrava-se no nível sócio-econômico categorizado como classe A e B1 segundo ABEP (55,7\%) e tinha uma renda familiar de 1,1 a 6 salários mínimos (55,8\%), sendo que $74,4 \%$ tinham nível superior completo de escolaridade.

Quanto às características laborais, a população estudada era composta de $44,8 \%$ professores, pesquisadores e profissionais liberais e $55,2 \%$ de profissionais técnico-administrativos. Entre variáveis relacionadas ao trabalho observou-se que mais da metade das pessoas tinham uma carga horária de 6 a 8 horas $(54,8 \%), 5,4 \%$ dos entrevistados consideravam o esforço despendido no trabalho pesado ou muito pesado e apenas 7,5\% classificaram-no como penoso e que lhe causava sofrimento. Definiram-se as variáveis referentes à posição adotada no trabalho a maior parte do tempo, reunindo às freqüências obtidas nas categorias "freqüentemente e sempre". Constatouse que $88,2 \%$ trabalhavam, freqüentemente ou sempre, sentados; $47,1 \%$, com movimentos de inclinação do tronco, 23,6\%, em pé, parados, e $17,3 \%$, carregando peso. Porém, 54\% nunca ou raramente adotavam a posição de trabalhar em pé caminhando (Tabela 1).

Observou-se que 75,9\% dos indivíduos da amostra não fumavam (Tabela 1). Constatou-se que $32,7 \%$ realizavam atividade física três vezes por semana com duração de no mínimo trinta minutos. Entre os indivíduos incluídos na análise $44,7 \%$ apresentavam IMC correspondente a sobrepeso e obesidade.

Na análise bruta das variáveis demográficas e sócio-econômicas observou-se que a dor lombar foi mais referida no sexo feminino $(p=0,06)$. De acordo com a idade, os resultados mostraram que a maior prevalência foi observada entre os indivíduos de 20 e 29 anos, e conforme aumentava a idade, verificou-se uma redução da prevalência de dor, encontrando-se associação estatisticamente significativa $(\mathrm{p}=0,01)$ (Tabela 1$)$. 
Prevalência, razões de prevalência (RP) com respectivos intervalos de confiança (IC95\%) e valores de p para dor lombar nos últimos três meses de acordo com variáveis demográficas, sócio-econômicas, hábitos de vida e ocupacionais. São Leopoldo, Rio Grande do Sul, Brasil, 2006

\begin{tabular}{|c|c|c|c|c|c|c|}
\hline Variável & $\mathrm{n}$ & $\%$ & Prevalência & RP & IC95\% & Valor de $p$ \\
\hline Sexo $(n=775)$ & & & & & & 0,06 * \\
\hline Masculino & 369 & 47,6 & 42,5 & 1,00 & - & \\
\hline Feminino & 406 & 54,2 & 49,3 & 1,16 & $0,99-1,35$ & \\
\hline Idades agrupadas em intervalos & & & & & & $0,01 * \star$ \\
\hline \multicolumn{7}{|l|}{ de 10 anos $(n=774)$} \\
\hline $20-29$ & 162 & 20,9 & 54,3 & 1,00 & - & \\
\hline $30-39$ & 255 & 32,9 & 48,8 & 0,90 & $0,74-1,09$ & \\
\hline $40-49$ & 244 & 31,5 & 38,8 & 0,72 & $0,58-0,88$ & \\
\hline $50-59$ & 113 & 14,6 & 43,4 & 0,80 & $0,39-1,07$ & \\
\hline Trabalho diário penoso $(n=773)$ & & & & & & 0,03 * \\
\hline Não & 715 & 92,5 & 44,9 & 1,00 & - & \\
\hline Sim & 58 & 7,5 & 59,6 & 1,33 & $1,06-1,67$ & \\
\hline Função no trabalho $(\mathrm{n}=756)$ & & & & & & 0,02 * \\
\hline Professor, pesquisador, profissional liberal & 339 & 44,8 & 41,7 & 1,00 & - & \\
\hline Técnico-administrativo & 417 & 55,2 & 50,2 & 1,20 & $1,03-1,41$ & \\
\hline Trabalha sentado $(n=768)$ & & & & & & 0,18 * \\
\hline Nunca e raramente & 91 & 11,8 & 39,6 & 1,00 & - & \\
\hline Freqüentemente e sempre & 677 & 88,2 & 47 & 1,19 & $0,91-1,55$ & \\
\hline Trabalha em pé caminhando $(n=750)$ & & & & & & 0,08 * \\
\hline Nunca e raramente & 405 & 54,0 & 43,7 & 1,00 & - & \\
\hline Freqüentemente e sempre & 345 & 46,0 & 50 & 1,14 & $0,98-1,33$ & \\
\hline Trabalha com inclinação do tronco $(n=770)$ & & & & & & 0,01 * \\
\hline Nunca & 63 & 8,2 & 34,9 & 1,00 & - & \\
\hline Raramente & 344 & 44,7 & 42,9 & 1,23 & $0,86-1,76$ & \\
\hline Freqüentemente e sempre & 363 & 47,1 & 51,5 & 1,48 & $1,04-2,10$ & \\
\hline Trabalha carregando peso $(n=772)$ & & & & & & 0,16 * \\
\hline Nunca & 185 & 24,0 & 41,1 & 1,00 & - & \\
\hline Raramente & 453 & 58,7 & 46,6 & 1,13 & $0,93-1,38$ & \\
\hline Freqüentemente e sempre & 134 & 17,3 & 51,9 & 1,26 & $1,00-1,60$ & \\
\hline Fumo $(n=771)$ & & & & & & 0,12 * \\
\hline Não fuma & 585 & 75,9 & 46,3 & 1,00 & - & \\
\hline Ex-fumante & 121 & 15,7 & 39,7 & 0,86 & $0,68-1,09$ & \\
\hline Fumante & 65 & 8,4 & 54,5 & 1,20 & $0,95-1,51$ & \\
\hline
\end{tabular}

* Teste do qui-quadrado;

** Teste de tendência linear.

As variáveis cor da pele observada, viver com companheiro, classe econômica, escolaridade e renda familiar per capita não estavam associadas ao desfecho.

Nas variáveis relacionadas ao trabalho foi observado que a prevalência da dor foi maior nos indivíduos classificados na categoria dos técnico-administrativos ( $p=0,02$ ), entre os que consideravam o trabalho diário penoso $(p=0,03)$ e entre aqueles que trabalhavam com inclinação do tronco $(p=0,01)$ (Tabela 1). Não foram observadas diferenças estatisticamente significativas quanto à carga diária no trabalho, esforço no trabalho, trabalha sentado, trabalha em pé caminhando, trabalha em pé parado e se carrega peso .

Não foram encontradas diferenças estatisticamente significativas em relação ao hábito de fumar, ao índice de massa corporal, a referir atividade física ou a freqüência de atividade física. 
Contudo, a variável fumo foi incluída no modelo por apresentar nível de significância de 0,12 (Tabela 1).

Na análise multivariável através de regressão de Poisson foram encontradas associações entre dor lombar nos últimos três meses em relação aos indivíduos de 40 a 49 anos (RP: 0,71; IC95\%: 0,58-0,88), naqueles que consideravam seu trabalho como penoso (RP: 1,36; IC95\%: 1,10-1,68) e nos fumantes (RP: 1,27; IC95\%: 1,00-1,62) (Tabela 2).

\section{Discussão}

Pelo fato de dor lombar ser considerado um sintoma, ao invés de uma doença, os estudos investigativos dependem muito das informações e recordações dos sujeitos participantes das pesquisas. Como as formas de coleta de dados têm variado nos diferentes estudos, as freqüências dos desfechos podem apresentar resultados diferentes conforme as definições adotadas. Assim sendo, optou-se por analisar a freqüência de dor lombar nos últimos três meses, embora se tenha descrito as prevalências relacionadas ao desfecho levando em consideração diferentes localizações, tempo de ocorrência e incapacidades.

A prevalência de dor lombar no último ano identificada nesta pesquisa deve ser considerada muito elevada e semelhante às encontradas em outros estudos 1,2,3,27,28,29,30. Numa revisão de literatura envolvendo o período de 1966 a 1988, a prevalência de dor lombar no último ano variou entre $22 \%$ e $65 \% 27$. Schneider et al. 28 investigaram a prevalência de dor nas costas em um estudo transversal com uma amostra representativa da população alemã trabalhadora e encontraram uma prevalência no ano de $60 \%$ de queixa de dor nas costas, resultado inferior ao de $71,5 \%$ encontrado neste estudo. Já em pesquisas realizadas entre trabalhadores houve uma variação de prevalência-ano de $52,1 \%$ no estudo transversal de Bejia et al. 29, onde foi investigada a presença de dor lombar em equipe hospitalar na Tunísia, a $74,6 \%$, no estudo transversal de Rugelj 30 com fisioterapeutas na Eslovênia. Poucos estudos utilizaram o principal ponto de corte da presente investigação, mas Thomas et al. 31 em coorte realizada com pacientes que já haviam participado de um estudo transversal, encontraram uma prevalência de $48 \%$ nos últimos três meses. Em estudo transversal, realizado nos Estados Unidos, incluindo população a partir de 18 anos, foi encontrada uma prevalência de $31 \%$ de dor lombar nos últimos três meses 32 .

No presente estudo verificou-se que as mulheres tiveram uma maior prevalência de dor lombar, concordando com outros estudos epidemiológicos como o de base populacional realizado na Turquia por Gilgil et al. 33 , o estudo transversal de Bejia et al. ${ }^{29}$, o estudo transversal

Tabela 2

Análise multivariável através de regressão de Poisson para dor lombar nos últimos três meses. São Leopoldo, Rio Grande do Sul, Brasil, 2006.

\begin{tabular}{|c|c|c|c|}
\hline Variável & RP & IC95\% & Valor de $p$ \\
\hline Sexo * & & & $0,05 * \star$ \\
\hline Masculino & 1,00 & - & \\
\hline Feminino & 1,16 & $1,00-1,36$ & \\
\hline 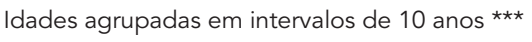 & & & 0,02 ** \\
\hline $20-29$ & 1,00 & - & \\
\hline $30-39$ & 0,90 & $0,75-1,09$ & \\
\hline $40-49$ & 0,71 & $0,58-0,88$ & \\
\hline $50-59$ & 0,80 & $0,62-1,03$ & \\
\hline Trabalho diário penoso \# & & & $0,01 \star \star$ \\
\hline Não & 1,00 & - & \\
\hline Sim & 1,36 & $1,10-1,68$ & \\
\hline
\end{tabular}

RP: razão de prevalência; IC95\%: intervalo de 95\% de confiança.

* Ajustado por sexo;

** Teste de Wald para heterogeneidade;

*** Ajustado por idade;

\# Ajustado para sexo e idade. 
de base populacional sobre dor lombar crônica realizado na zona urbana de Pelotas, Rio Grande do Sul, Brasil, por Silva et al. 18 e o estudo populacional de Papageorgiou et al. 14 no Reino Unido. As mulheres apresentaram maiores queixas de dor possivelmente em decorrência de algumas características anátomo-funcionais, tais como menor massa óssea, menor resistência muscular e articulações mais instáveis, e em função de sua força muscular ser $30 \%$ menor do que a dos homens, em média 34.

No presente estudo encontrou-se a tendência de dor lombar inversa ao aumento da idade. Uma justificativa plausível para esse achado seria o fato de os indivíduos com mais idade exercerem mais cargos de chefia e tarefas administrativas, não sendo submetidos à uma sobrecarga na coluna. Striebel 35 referiu em estudo com fisioterapeutas neurológicos em Porto Alegre, Rio Grande do Sul, Brasil, mostrou que as pessoas mais velhas ocupavam mais cargos de chefia, não estando tão expostas às exigências físicas do trabalho e, conseqüentemente, ao surgimento do sintoma. Contudo, em outras pesquisas constatou-se um aumento da prevalência com o decorrer da idade $1,2,3,14,18,36$.

Considerar que o trabalho diário foi classificado como penoso e que causava sofrimento permaneceu associado à dor lombar nos últimos três meses, ajustado por sexo e idade. Esse achado esteve de acordo com outros estudos indicando associação entre fatores psicológicos e estresse no trabalho e a ocorrência de dor lombar $10,28,37$.

Desordens músculo-esqueléticas da coluna lombar são importantes problemas de saúde pública, assim deve-se formular estratégias de intervenção para o controle desta morbidade. Segundo o National Research Council dos Estados Unidos 16, desordens músculo-esqueléticas são provocadas pela exposição no trabalho e também por fatores externos e individuais. A Organização Mundial da Saúde denomina de "condições relacionadas ao trabalho (work-related conditions)" os fatores não relacionados à atividade laboral como, por exemplo, aspectos físicos e sociais, há- bitos de vida, características individuais e predisposição genética que podem estar relacionados ao surgimento dessas desordens, além das exposições no trabalho.

Por outro lado, Facchini ${ }^{38}$, chamando atenção para formulações teóricas da epidemiologia ancorada no modelo de determinação social da doença, afirma que cargas de trabalho, conceito originalmente proposto por Laurell \& Noriega 39 para análise do impacto no processo de trabalho sobre a saúde do trabalhador, são exigências ou demandas que o processo laboral impõe e que geram as particularidades do desgaste do trabalhador 40 . Sabe-se que as pessoas passam grande parte do tempo trabalhando e submetidas a exigências e demandas físicas e psíquicas capazes de produzir desgaste. Observa-se que o processo de trabalho pode ser, e em geral é, o fator predominante e certamente pode provocar ou agravar um quadro de desordem músculo-esquelética ou ser o agente causador de várias morbidades associadas.

No presente estudo pode-se observar elevadas prevalências de dor lombar. Contudo, apesar da elevada prevalência na população estudada e de ter causado dificuldade no desempenho das atividades laborais, a dor lombar não foi incapacitante a ponto de causar repercussões como o aumento do absenteísmo ou um maior número de consultas médicas. Apesar de 29,9\% dos trabalhadores referirem ter tido dificuldades para realizar suas atividades de vida diária ou no trabalho por dor lombar, o presente estudo detectou um absenteísmo muito baixo.

Posturas inadequadas, movimentos repetitivos, tensão muscular e estresse são sabidamente alguns dos fatores envolvidos na gênese da dor lombar, especialmente entre docentes e profissionais técnico-administrativos. É importante não somente adotar medidas efetivas para o diagnóstico e tratamento das lesões músculo-esqueléticas nos trabalhadores, mas principalmente identificar as possíveis causas, favorecendo o planejamento de programas de intervenção e a formulação de propostas de prevenção e promoção da saúde. 


\section{Resumo}

Com o objetivo de determinar a prevalência de dor lombar nos últimos três meses e fatores associados foi realizado um estudo transversal, com indivíduos de ambos os sexos, de 20 a 59 anos titulares do plano de saúde da COOPERSINOS da Universidade do Vale do Rio dos Sinos. Foram utilizados questionários autoaplicados incluindo questões demográficas, sócio-econômicas, ocupacionais e hábitos de vida. Entre os 775 indivíduos a prevalência de dor lombar no último ano foi de 52,8\% (IC95\%: 49,3-56,3) e nos últimos três meses foi de 46\% (IC95\%: 42,5-49,5). Através de regressão de Poisson foram encontradas associações para dor lombar nos últimos três meses nos indivíduos de 40 a 49 anos, naqueles que consideravam seu trabalho penoso e nos fumantes. Apesar da elevada prevalência de dor lombar na população estudada e de ter provocado dificuldades no desempenho das atividades laborais, a condição não foi incapacitante. Mesmo que 29,9\% dos trabalhadores tenham referido dificuldades para realizar suas atividades de vida diária ou no trabalho por dor lombar, detectou-se absenteísmo muito baixo.

Dor Lombar; Planos de Pré-Pagamento em Saúde; Prevalência

\section{Referências}

1. Barros MBA, Chester LG, Carandina L, Dalla Torre G. Desigualdades sociais na prevalência de doenças crônicas no Brasil, PNAD-2003. Ciênc Saúde Coletiva 2006; 11:911-26.

2. Deyo RA, Weinstein JN. Low back pain. N Engl J Med 2001; 344:363-70.

3. Engstrom JW. Back and neck pain. In: Kasper DL, Fauci AS, Longo DL, Braunwald E, Hauser SL, Jameson JL, editors. Principles of internal medicine. New York: McGraw-Hill; 2005.p. 84-104.

4. Mendes R. O impacto dos efeitos da ocupação sobre a saúde de trabalhadores: I. Morbidade. Rev Saúde Pública 1988; 22:311-26.

5. Deyo R. Low back pain. Sci Am 1998; 279:48-53.

6. Andersson GBJ. Epidemiologic aspects on lowback pain in industry. Spine 1981; 6:53-60.

7. Ehrlich GE. Low back pain. Bull World Health Organ 2003; 81:671-6.

8. Kääriä S, Kaila-Kangas L, Kirjonen J, Riihimäki H, Luukonen R, Leino-Arjas P. Low back pain, work absenteeism, chronic back disorders, and clinical findings in the low back as predictors of hospitalization due to low back disorders: a 28 -year followup of industrial employees. Spine 2005; 30:1211-8.

\section{Colaboradores}

M. G. Matos supervisionou o trabalho de campo e participou da análise dos dados e da redação do manuscrito. E. A. Hennington e J. S. Dias-da-Costa participaram da análise dos dados, redação e revisão do manuscrito. A. L. Hoefel contribuiu na análise dos dados, redação do manuscrito.

\section{Agradecimentos}

Financiamento: Cooperativa dos Usuários de Serviços de Saúde do Vale do Rio dos Sinos e Universidade do Vale do Rio dos Sinos.
9. Biering-Sorensen F. A prospective study of low back pain in a general population. Scand J Rehab Med 1983; 15:71-9.

10. Andersson GBJ. Epidemiological features of chronic low-back pain. Lancet 1999; 354:581-5.

11. Calliet R. Lombalgias: síndromes dolorosas. São Paulo: Editora Manole; 2001.

12. Meirelles ES. Lombalgia: como diagnosticar e tratar. RBM Rev Bras Med 2003; 60:111-9.

13. Cox JM. Dor lombar: mecanismo, diagnóstico e tratamento. São Paulo: Editora Manole; 2002.

14. Papageorgiou AC, Croft PR, Ferry S, Jayson MIV, Silman AJ. Estimating the prevalence of low back pain in the general population: evidence from the South Manchester back pain survey. Spine 1995; 20:1889-94.

15. Soibelman M, Schenatto CB, Restelli VG. Dor lombar. In: Duncan B, Schmidt MI, Giugliane ERJ, organizadores. Medicina ambulatorial: condutas de atenção primária baseadas em evidências. $3 \underline{a}$ Ed. Porto Alegre: Editora Artmed; 2004. p. 1218-31.

16. National Research Council. Musculoskeletal disorder and the workplace. Washington DC: National Academies Press: 2001. 
17. Cole DC, Ibrahim SA, Shannon HS, Scott F, Eyles J. Work correlates of back problems and activity restriction due to musculoskeletal disorders in the Canadian national population health survey (NPHS) 1994-5 data. Occup Environ Med 2001; 58:728-34.

18. Silva MC, Fassa AG, Valle NCJ. Dor lombar crônica em uma população adulta do sul do Brasil: prevalência e fatores associados. Cad Saúde Pública 2004; 20:377-85.

19. Vogt MS. Prevalência e severidade da dor cervical e lombar nos servidores técnico-administrativos da Universidade Federal de Santa Maria [Dissertação Mestrado]. Florianópolis: Universidade Federal de Santa Catarina; 2000.

20. Kuorinka I, Jonsson B, Kilbom A, Vinterberg H, Biering-Sorensen F, Anderson G, et al. Standardized Nordic questionnaires for analyses of musculoskeletal symptoms. Appl Ergon 1987; 18:233-7.

21. Barros ENC, Alexandre NMC. Cross-cultural adaptation of the Nordic musculoskeletal questionnaire. Int Nurs Rev 2003; 50:101-8.

22. World Health Organization. Obesity: preventing and managing the global epidemic. Report of a WHO Consultation on Obesity. Geneva: World Health Organization; 1998.

23. Matsudo V. Physical activity: passport for health. World Health Report 1997; 50:16-7.

24. Altman DG. Practical statistics for medical research. London: Chapman \& Hall; 1997.

25. Victora CG, Huttly SR, Fuchs SC, Olinto MTA. The role of conceptual frameworks in epidemiological analysis: a hierarchical approach. Int J Epidemiol 1997; 26:224-7.

26. Barros AJ, Hirakata VN. Alternatives for logistic regression in cross-sectional studies: an empirical comparison of models that directly estimate the prevalence ratio. BMC Med Res Methodol 2003; 3:21.

27. Walker BF. The prevalence of low back pain: a systematic review of the literature from 1966 to 1998. J Spinal Disord 2000; 13:205-17.

28. Schneider S, Schmitt H, Zoller S, Schiltenwolf M. Work place stress, lifestyle and social factors as correlates of back pain: a representative study of the German working population. Int Arch Occup Environ Health 2005; 78:253-69.
29 Bejia I, Younes M, Jamila HB, Khalfallah T, Salem $\mathrm{KB}$, Touzi M, et al. Prevalence end factors associated to low back pain among hospital staff. Joint Bone Spine 2004; 72:254-9.

30. Rugelj D. Low back pain and another work-related musculoskeletal problems among physiotherapists. Appl Ergon 2003; 34:635-9.

31. Thomas E, Silman AJ, Croft PR, Papageorgiou AC Macfarlane GJ. Predicting who develops chronic low back pain in primary care: a prospective study. BMJ 1999; 318:1662-7.

32. Strine TW, Hootman JM. US national prevalence and correlates of low back pain and neck pain among adults. Arthritis Rheum 2007; 57:656-65.

33. Gilgil E, Kaçar C, Bütün B, Tuncer T, Sevgi U, Çinar $\mathrm{Y}$, et al. Prevalence of low back pain in a developing urban sitting. Spine 2005; 30:1093-8.

34. Nascimento N, Moraes R. Fisioterapia nas empresas. Rio de Janeiro: Taba Cultural; 2000.

35. Striebel VLW. Perfil macroergonômico dos fisioterapeutas neurológicos de Porto Alegre [Dissertação de Mestrado]. Porto Alegre: Curso de Pós-graduação em Engenharia de Produção, Universidade Federal do Rio Grande do Sul; 2004.

36. Friedrich M, Cermak T, Heiller I. Spinal troubles in sewage workers: epidemiological data and work disability due to low back pain. Int Arch Occup Environ Health 2000; 73:245-54.

37. Polatin PB, Kinney RK, Gatchel RJ, Lillo E, Mayer TG. Psychiatric illness and chronic back pain. The mind and the spine which goes first? Spine 1993; 18:66-71.

38. Facchini LA. Uma contribuição da epidemiologia: o modelo da determinação social aplicado à saúde do trabalhador. In: Rocha LE, Rigotto RM, Buschinelli JTP, organizadores. Isto é trabalho de gente? Vida, doença e trabalho no Brasil. Petrópolis: Editora Vozes; 1993. p. 178-86.

39. Laurel AC, Noriega M. Processo de produção e saúde: trabalho e desgaste operário. São Paulo: Editora Hucitec; 1989.

40. Facchini LA. Processo de trabajo, cambio tecnológico y desgaste obrero [Disertación de Maestría]. México DF: Universidad Autónoma Metropolitana-Xochimilco; 1986.

Recebido em 17/Jan/2007

Versão final reapresentada em 16/Jan/2008

Aprovado em 15/Fev/2008 